\title{
Ciclo de mejora docente en la titulación de Ingeniería Mecánica: hacia un conocimiento real de las estructuras
}

\author{
BEATRIZ HORTIgón FuENTES \\ DEPARTAMENTO DE MECÁNICA DE MEDIOS \\ CONTINUOS Y TEORÍA DE ESTRUCTURAS \\ ESCUELA POLITÉCNICA SUPERIOR \\ UNIVERSIDAD DE SEVILLA \\ bhortigon@us.es \\ D.0.I.: http://dx.doi.org/10.12795/JDU.2018.i01.55 \\ Pp.: 979-995
}

\section{Resumen}

La presente comunicación narra la experiencia del Ciclo de Mejora llevado a cabo en el área de Mecánica de Medios Continuos y Teoría de Estructuras sobre el tema del cálculo de deformaciones en diferentes tipologías de elementos estructurales. En la misma se detalla de manera estructurada el modelo metodológico utilizado y la secuencia de actividades llevadas a cabo, un breve diario de las sesiones y un estudio final de la evolución del aprendizaje de los estudiantes durante el ciclo. Las sesiones han sido grabadas para su análisis dentro del Proyecto de Excelencia del Plan Estatal 2013-2016 titulado “La Formación Docente del Profesorado Universitario. Progresos y Obstáculos de los Participantes en un Programa Basado en Ciclos de Mejora de su Práctica". 
Palabras Clave: Innovación docente, áreas de Arquitectura e Ingeniería, Resistencia de Materiales, Estructuras, Aprendizaje por resolución de problemas, Trabajo grupal.

\section{Contexto}

El ciclo de innovación docente para el curso 2018/19 se ha realizado en el marco de la asignatura "Resistencia de Materiales. Estructuras", asignatura troncal del segundo curso del Grado en Ingeniería Mecánica (Plan 2010). Se ha trabajado sobre los mismos temas de aprendizaje que durante el curso anterior, el cálculo de deformaciones en vigas isostáticas e hiperestáticas sometidas a flexión, con el mismo número de horas (6h divididas en 3 clases de $2 \mathrm{~h}$ ) y el mismo sistema, basado en el trabajo del alumno en el aula mediante Hojas de Trabajo con preguntas encadenadas en el inicio para el entendimiento de los conceptos básicos, y ejercicios posteriores de carácter procedimental.

La decisión de incidir sobre el ciclo de mejora del curso anterior tiene como objetivo principal el mejorar diversos aspectos de metodología y contenido, más ajustados al tiempo y a los objetivos de aprendizaje que entiendo debe alcanzar el alumno tras su realización.

La experiencia se ha realizado en un grupo compuesto aproximadamente de 70 estudiantes, siendo la asistencia media durante el ciclo de 55 alumnos. Las sesiones se han desarrollado todas en un mismo aula, diseñada para dar clases teóricas, lo que ha dificultado en cierta manera el trabajo grupal. 


\section{Principios didácticos argumentados}

A nivel organizativo, el diseño del ciclo se ha realizado desde la creencia de que el trabajo en grupo, además de favorecer el aprendizaje de los estudiantes, mejora también su capacidad de relacionarse con otros individuos a nivel intelectual, preparándolos para su futura carrera profesional.

A nivel de contenidos se han planteado problemas potentes relacionados con los conceptos realmente importantes de los temas, de manera que los estudiantes los vayan resolviendo de manera gradual, sin explicación previa alguna por parte del profesor. Es importante que los estudiantes construyan su conocimiento desde sus propios razonamientos y desde sus propios errores.

Respecto a la evaluación del aprendizaje se considera importante conocer la evolución de cada estudiante tras las sesiones, mediante la contestación de un cuestionario antes y después del ciclo.

\section{Mapa de contenidos y preguntas clave}

En la Figura 1 se presenta el mapa de contenidos junto con las preguntas-problemas clave que han constituido el cuestionario inicial y final realizado por el alumnado.

El mapa de contenidos se plantea desde dos grandes conceptos que son: la diferenciación entre los dos tipos de estructuras existentes (isostáticas e hiperestáticas) desde el punto de vista de su capacidad de deformación y los diferentes factores que influyen en la misma. El conocimiento de los mismos es fundamental para optimizar el diseño a fin de crear estructuras armónicas y ajustadas a precios de mercado. A partir de estos contenidos conceptuales principales se desarrollan los diferentes procedimientos para el cálculo de dichas deformaciones según Gere (2009), a los que se añaden conceptos de segundo orden que serán necesarios para su desarrollo. 
¿Qué diferencias hay entre una estructura isostática y una hiperestática?

¿Cómo se determina el grado de hiperestaticidad de una estructura?

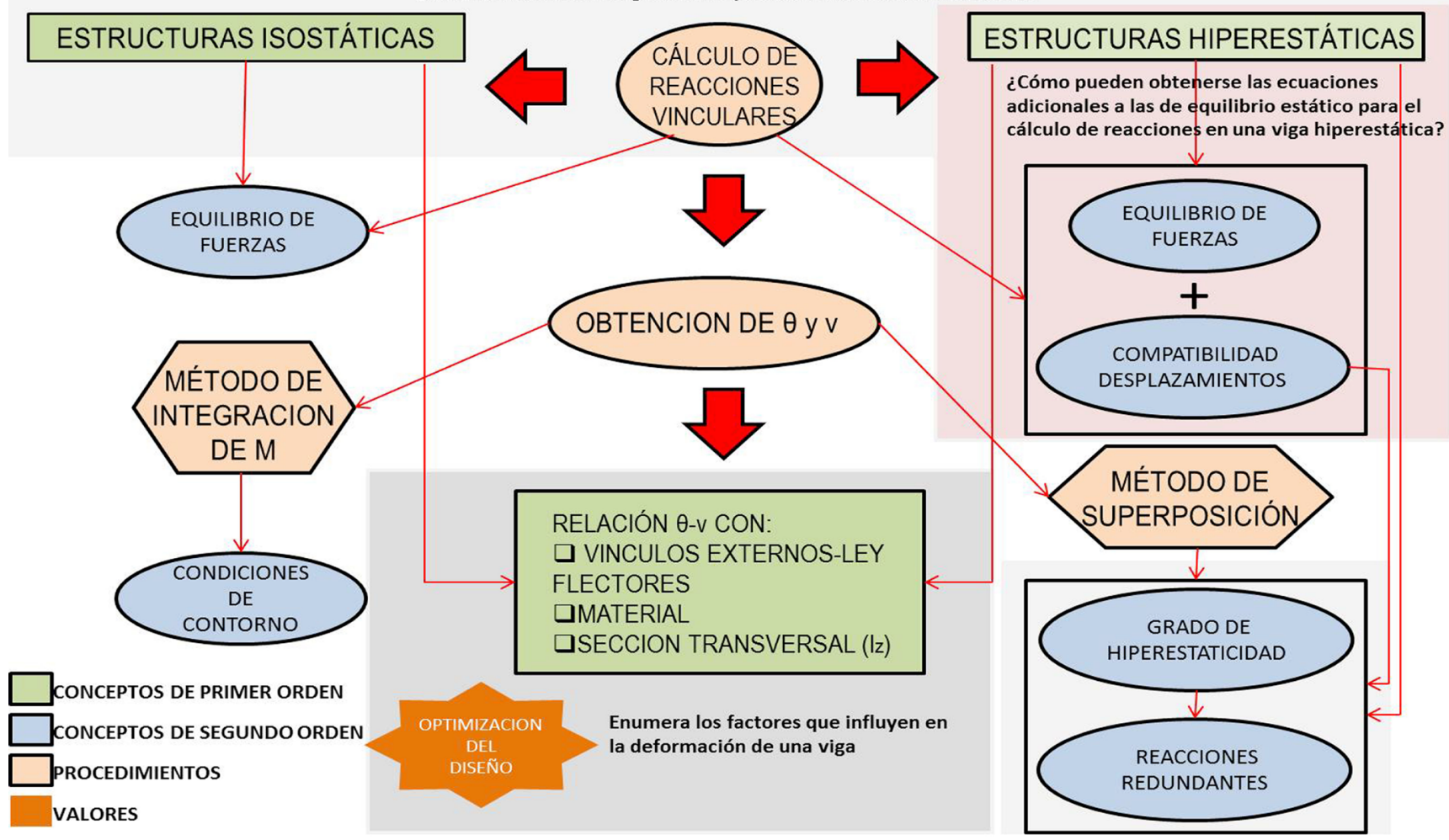

Figura 1. Mapa de contenidos y preguntas clave

Jornadas de Formación e Innovación Docente del Profesorado | № 1 (2018)

cc (†) Esta obra se distribuye con la licencia Creative Commons

Internacional (CC BY-NC-ND 4.0.) 


\section{Modelo metodológico argumentado y secuencia de actividades}

El ciclo se ha planteado mediante talleres de trabajo de tipo conceptual o procedimental. Para cada taller se han preparado Hojas de Trabajo basadas en preguntas encadenadas, atendiendo a la metodología descrita en Finkel (2005). En este aspecto general, no se han realizado cambios respecto al ciclo de mejora del curso pasado.

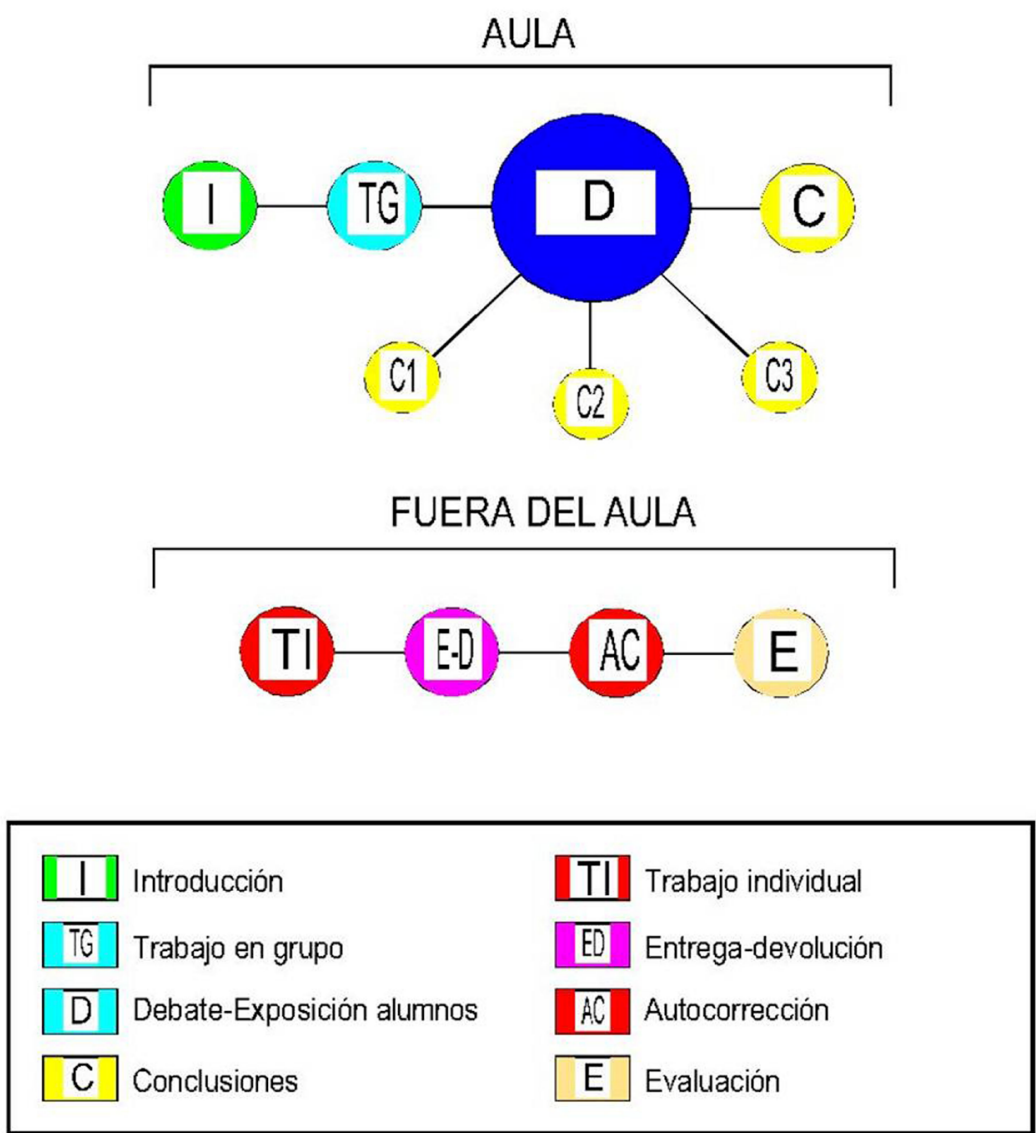

Figura 2. Modelo metodológico

El modelo metodológico utilizado para las tres clases sigue la misma secuencia. La clase se inicia con una breve introducción por mi parte, la cual será un poco más extensa

Jornadas de Formación e Innovación Docente del Profesorado | № 1 (2018)

Esta obra se distribuye con la licencia Creative Commons Reconocimiento-NoComercial-SinObraDerivada $\quad 4.0$ Internacional (CC BY-NC-ND 4.0.) 
en la primera jornada para explicar al alumnado la forma de trabajo durante el ciclo. Posteriormente se hace entrega a los estudiantes de la Hoja de Trabajo correspondiente a la sesión, en la que se incluye un texto inicial con todas las instrucciones de la actividad (estructuración completa de la clase, tamaño de los grupos, duración de los ejercicios, etc.). Una vez que han leído el documento y, preguntado cualquier duda, se pasa a la realización de la actividad en grupos de dos o tres estudiantes, con un posterior debate-exposición de ideas para cada una de las preguntas sobre las que se irán extrayendo conclusiones parciales. Al final del taller se realiza un resumen por mi parte de todas estas conclusiones.

Respecto los contenidos de las Hojas de Trabajo, la experiencia del año anterior me llevó a concluir que el tiempo planteado para los ejercicios estaba excesivamente ajustado, por lo que para este curso he reajustado de inicio el contenido de los mismos para tratar de tener un poco más de holgura.

El modelo descrito se ha seguido en las tres clases, variando la duración de las fases de trabajo de los estudiantes y el debate posterior en función de la magnitud y tipología de las actividades planteadas en cada una de ellas.

Como innovación al ciclo del curso pasado, este año he introducido un trabajo voluntario que podrán entregarme todos los estudiantes que hayan asistido al menos a dos de las tres clases del ciclo, y que supondrá un 10\% de la nota final.

Dicho trabajo se realizará de manera individual y consistirá en la resolución de ejercicios similares a los realizados en clase. La secuencia de este trabajo fuera del aula será la siguiente: tras una primera entrega del ejercicio por parte del estudiante, pasados unos días se publicará la 
solución en la webCT para que el estudiante realice una autocorrección de su trabajo y vuelvan a hacer una segunda entrega de su ejercicio con dicha autocorrección. La actividad evaluable será ésta segunda, no la primera. Con ello pretendo dar valor al aprendizaje basado en la detección de errores, siguiendo la metodología expuesta por Xavier Domènech en las IV Jornadas de Formación e Innovación Docente de la Universidad de Sevilla.

La secuencia de actividades prevista para el ciclo es la siguiente:

\begin{tabular}{|l|l|l|l|}
\hline SESIÓN 1 (1h 55' & aproximadamente) \\
\hline & & $\begin{array}{l}\text { Breve explicación de la sistemática del Taller } \\
\text { I }\end{array}$ & $1^{\prime}$ \\
\hline 5' & $\begin{array}{l}\text { Entrega de la Hoja de Trabajo 1, en la que se } \\
\text { explica detalladamente cómo deben organi- } \\
\text { zarse, la temática sobre la que se va a traba- } \\
\text { jar y el tiempo disponible para la resolución } \\
\text { de las preguntas }\end{array}$ & $2^{\prime}$ \\
\hline \multirow{2}{*}{ TG } & $\begin{array}{l}\text { Organización en grupos de 2 alumnos de } \\
\text { manera autónoma }\end{array}$ & $2^{\prime}$ \\
\hline $15^{\prime}$ & $\begin{array}{l}\text { Resolución en grupo del Apartado A de la } \\
\text { Hoja de Trabajo 1, en la que se trabajará so- } \\
\text { bre los siguientes problemas o preguntas } \\
\text { claves (asociadas a Contenidos Conceptua- } \\
\text { les): Enumera los factores que influyen en la } \\
\text { deformación de una viga. } \\
\text { Para que los estudiantes lleguen a respon- } \\
\text { der a estas preguntas se han planteado 5 } \\
\text { preguntas encadenadas. }\end{array}$ & 15' \\
\hline 20' & $\begin{array}{l}\text { Exposición y debate de cada una de las pre- } \\
\text { guntas. Se pretende que las conclusiones } \\
\text { obtenidas de cada pregunta se vayan enca- } \\
\text { denando hasta llegar al entendimiento de } \\
\text { los problemas principales. Todas las pregun- } \\
\text { tas de la Hoja son muy intuitivas por lo que } \\
\text { se espera que ellos solos puedan deducir } \\
\text { las respuestas de estos problemas. }\end{array}$ & 20' \\
\hline
\end{tabular}

Jornadas de Formación e Innovación Docente del Profesorado | № 1 (2018) Esta obra se distribuye con la licencia Creative Commons Reconocimiento-NoComercial-SinObraDerivada Internacional (CC BY-NC-ND 4.0.) 


\begin{tabular}{|c|c|c|c|}
\hline C & $10^{\prime}$ & $\begin{array}{l}\text { Las conclusiones finales de esta primera } \\
\text { parte se han planteado con un poco más de } \\
\text { tiempo, ya que la idea es explicarles la de- } \\
\text { mostración de las fórmulas que permiten } \\
\text { calcular deformaciones en vigas, en la que } \\
\text { se darán cuenta de que en la misma partici- } \\
\text { pan todos los factores que antes han dedu- } \\
\text { cido con su razonamiento. }\end{array}$ & $10^{-}$ \\
\hline & 5 & Descanso & 5 \\
\hline TG & $35^{\circ}$ & $\begin{array}{l}\text { Resolución en grupo del Apartado } 2 \text { de la } \\
\text { Hoja de Trabajo 1, en la que se trabajará el } \\
\text { método de integración del momento flector } \\
\text { para el cálculo de deformaciones en vigas } \\
\text { isostáticas. Esta parte tendrá, por tanto, ca- } \\
\text { rácter procedimental }\end{array}$ & $35^{\circ}$ \\
\hline D & $20^{-}$ & $\begin{array}{l}\text { Exposición y debate. El objetivo es que ha- } \\
\text { yan sabido plantear las constantes de in- } \\
\text { tegración en función de las coacciones al } \\
\text { movimiento en los vínculos externos }\end{array}$ & $20^{-}$ \\
\hline C & 5 & $\begin{array}{l}\text { Conclusiones finales/Entrega actividad } \\
\text { individual }\end{array}$ & 5 \\
\hline
\end{tabular}

\begin{tabular}{|c|c|c|c|}
\hline \multicolumn{4}{|c|}{ SESIÓN 2 (1h $55^{\circ}$ aprox) } \\
\hline I & $3^{-}$ & $\begin{array}{l}\text { Entrega de la Hoja de Trabajo } 2 \text {. Al conocer } \\
\text { ya los estudiantes la sistemática de trabajo } \\
\text { se espera que no tarden mucho en organi- } \\
\text { zarse con el compañero/a del día anterior y } \\
\text { repartirles la actividad. }\end{array}$ & $3^{\prime}$ \\
\hline TG & $15^{\prime}$ & $\begin{array}{l}\text { Resolución en grupo del Apartado } 2 \text { de } \\
\text { la Hoja de Trabajo 2, en la que se traba- } \\
\text { jará sobre sobre los siguientes problemas } \\
\text { o preguntas claves (asociadas a Conteni- } \\
\text { dos Conceptuales): ¿Qué diferencias hay } \\
\text { entre una estructura isostática y una hi- } \\
\text { perestática? ¿Cómo se determina el grado } \\
\text { de hiperestaticidad de una estructura? } \\
\text { ¿Cómo pueden obtenerse las ecuaciones } \\
\text { adicionales a las de equilibrio estátatico } \\
\text { para el cálculo de reacciones en una viga } \\
\text { hiperestática? }\end{array}$ & $15^{\prime}$ \\
\hline D & $20^{\prime}$ & Exposición y debate & $20^{\prime}$ \\
\hline C & $10^{\prime}$ & Conclusiones finales & $10^{-}$ \\
\hline
\end{tabular}




\begin{tabular}{|l|l|l|l|}
\hline & $5^{\prime}$ & Descanso & 5 \\
\hline TG & $55^{\prime}$ & $\begin{array}{l}\text { Resolución en grupo del Apartado 2 de } \\
\text { la Hoja de Trabajo 2. Tendrá carácter } \\
\text { procedimental. }\end{array}$ & $55^{\prime}$ \\
\hline
\end{tabular}

\section{SESIÓN 3 (1h 50 aprox)}

\begin{tabular}{|c|c|c|c|}
\hline D & $55^{\circ}$ & $\begin{array}{l}\text { Exposición y Debate sobre el Apartado } 2 \text { de la } \\
\text { Hoja de Trabajo 2. Este ejercicio requiere lle- } \\
\text { var una sistemática muy precisa y ordenada } \\
\text { por lo que el ejercicio está planteado median- } \\
\text { tes preguntas secuenciales asociadas a cada } \\
\text { uno de los pasos que deben ir dando. Iremos } \\
\text { realizando el ejercicio paso a paso afianzando } \\
\text { conceptos. }\end{array}$ & $55^{\prime}$ \\
\hline \multirow[t]{4}{*}{ C } & 5 & Conclusiones finales & 5 \\
\hline & $10^{-}$ & Descanso & $10^{-}$ \\
\hline & $20^{\prime}$ & $\begin{array}{l}\text { Presentación del mapa de contenidos como } \\
\text { resumen de ambos temas/Entrega actividad } \\
\text { individual }\end{array}$ & $20^{\prime}$ \\
\hline & $20^{\prime}$ & $\begin{array}{l}\text { Resolución de Cuestionario de Preguntas Cla- } \\
\text { ves por el alumnado }\end{array}$ & $20^{\prime}$ \\
\hline
\end{tabular}

\section{Relato resumido de las sesiones}

\section{Primera sesión.}

Al llegar al aula le pido a dos alumnos que repartan la Hoja de Trabajo 1. A pesar de no ser necesario, ya que todas las instrucciones están escritas en la misma, les explico brevemente la sistemática y el esquema de la clase. Entre el reparto de la documentación y esta explicación se pierden aproximadamente 15 minutos, por lo que mi programación se retrasa desde el primer momento.

Sorprendentemente se ponen a trabajar en el más absoluto silencio, la gran mayoría de ellos discutiendo y compartiendo impresiones con los compañeros. Se agrupan 
conforme están ubicados en el aula, trabajando en grupos de dos o tres personas. Algunos alumnos, los menos, comienzan a trabajar solos. Trato de que se unan a algún grupo pero hacen caso omiso. Tampoco le doy la mayor importancia.

La verdad es que es un gusto verlos trabajar. Voy paseando para ver cómo avanzan y me acerco a ayudar a los más despistados. Empiezan a reclamarme por aquí y por alli y trato de atenderlos a todos de la mejor manera posible sin tampoco resolverles las cuestiones, únicamente guiándolos por el camino de su razonamiento para que alcancen los objetivos deseados.

Debido al gran número de alumnos con el que trabajo, me doy cuenta de que el tiempo previsto para la primera actividad es escaso ya que me es imposible atender a todos los que me reclaman en ese período. Sin embargo, decido prolongar dicho tiempo para que todos tengan encauzada al menos la actividad.

Durante el período de exposición tengo que tirar de la lista para sacarlos a la pizarra ya que ninguno se ofrece voluntario. Realmente el debate resulta muy productivo y desde los pupitres sí que participan y corrigen o apuntan al compañero que está en la pizarra. A todos les pregunto su nombre para tener un trato más cercano, creo que esto les ayuda a tener más confianza cuando realizan sus intervenciones.

Teniendo previsto para esta primera clase la realización de la segunda actividad para posterior debate en la clase siguiente, al final la clase termina empezando con ella.

Jornadas de Formación e Innovación Docente del Profesorado | № 1 (2018) Esta obra se distribuye con la licencia Creative Commons 
Segunda sesión.

La segunda clase continúa con la misma dinámica que la primera. Al comenzar con una actividad de carácter procedimental los noto muy inseguros con los planteamientos iniciales, tienen miedo a equivocarse. Los tranquilizo diciéndoles que no pasa nada por errar, que es parte del aprendizaje.

Con tanta cantidad de alumnos observo que algunos de ellos van sobrados en sus razonamientos, mientras que otros no saben ni por dónde empezar. Entiendo que debo ayudarlos pero no me resulta agradable ver que tengo a alumnos aburridos en clase.

La segunda exposición es más distendida, parece que han perdido el miedo, apareciendo algún voluntario para salir a la pizarra.

Sigo aumentando el tiempo de retraso, veo que no voy a terminar en las 6 horas previstas.

Tercera sesión y cuarta sesión

Tras la tercera sesión con la misma dinámica, debo alargar el ciclo una clase más. En esta última clase me comentan que están ya un poco cansados, me río y les comento que lógicamente trabajar cansa pero que se darán cuenta pronto de que han aprendido de verdad.

Curiosamente durante las clases magistrales ni ellos ni yo aguantamos las dos horas. Normalmente tenemos pactado el no hacer descanso y dar la clase del tirón con una duración aproximada de $1 \mathrm{~h} 45^{\circ}$. Con esta metodología de trabajo en el aula nadie está cansado, agotamos las dos horas e incluso algunos siguen trabajando cuando me marcho.

Jornadas de Formación e Innovación Docente del Profesorado | № 1 (2018) Esta obra se distribuye con la licencia Creative Commons 
En general, la experiencia ha sido muy positiva. A pesar de las dificultades de trabajar en un aula preparada para clases teóricas y con un grupo muy numeroso, la mayoría de los alumnos se han implicado mucho y creo que se han dado cuenta del trabajo previo que conlleva para el profesor aplicar esta metodología y de su utilidad.

\section{Evaluación del aprendizaje de los estudiantes}

A continuación se presentan las escaleras de aprendizaje resultado de los cuestionarios realizados por los alumnos antes y después del ciclo. Las preguntas de este cuestionario son las incluidas en el mapa de contenidos (Figura 2).

Pregunta 1. Enumera los factores que influyen en la deformación de una viga a flexión

Concediendo la misma importancia a los cuatro factores que supondrían la respuesta correcta, se observa una evolución del alumnado en cuanto al conocimiento de todos ellos, con un porcentaje inicial del $0 \%$ y uno final del $19,44 \%$. Se puede afirmar que prácticamente la mitad de los estudiantes ha alcanzado un nivel medio de aprendizaje $(44,44 \%)$, alcanzando un nivel óptimo (conocimiento de 3 ó 4 factores) un 24.99\%.

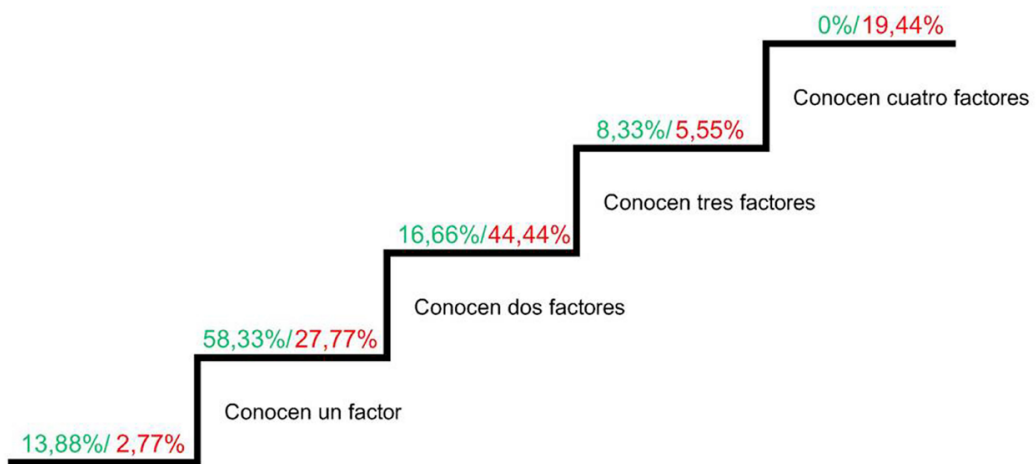

Figura 3. Escalera de aprendizaje pregunta 1

Jornadas de Formación e Innovación Docente del Profesorado | № 1 (2018) Esta obra se distribuye con la licencia Creative Commons 
Pregunta 2. ¿Qué diferencia hay entre una estructura isostática y una hiperestática?

En esta cuestión prácticamente todos los estudiantes han alcanzado el nivel óptimo de aprendizaje. Conviene mencionar que sobre esta pregunta se habían hecho comentarios en temas previos por lo que más de un tercio del alumnado (36,11\%) tenían asimilado el concepto antes de comenzar el ciclo. Tras su realización, un altísimo porcentaje de la clase $(88,88 \%)$ ha conseguido comprender la diferencia entre ambos tipos de estructuras.

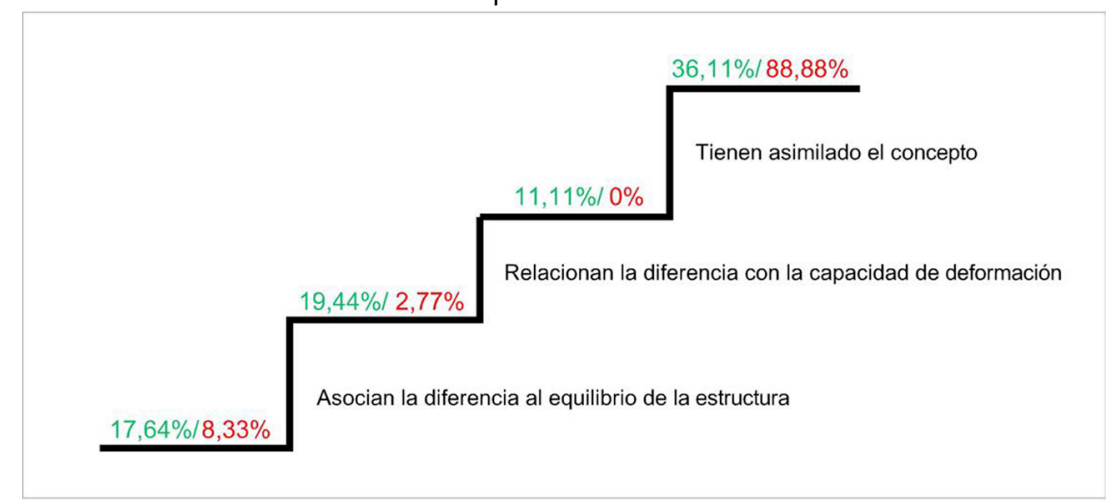

Figura 4. Escalera de aprendizaje pregunta 2

Pregunta 3. ¿Cómo se determina el grado de hiperestaticidad de una estructura?

En esta pregunta se partía de un nivel de conocimiento cero del $58,33 \%$, aunque también de un gran porcentaje de estudiantes con entendimiento del concepto (30,55\%). El resultado final arroja que la gran mayoría de alumnos situados en el nivel inferior han evolucionado hasta responder correctamente, quedando únicamente un $8,57 \%$ sin responder a la pregunta. Cabe destacar que en la escalera final desaparecen los escalones intermedios iniciales, lo que implica un claro entendimiento de su contenido por parte de los estudiantes.

Jornadas de Formación e Innovación Docente del Profesorado | № 1 (2018) Esta obra se distribuye con la licencia Creative Commons 
Lo asocian a la relación entre $n^{\circ}$ ecuaciones e incógnitas pero no conocen dicha relación

Figura 5. Escalera de aprendizaje pregunta 3

Pregunta 4. ¿Cómo pueden obtenerse las ecuaciones adicionales a las de equilibrio estático para el cálculo de reacciones en una viga hiperestática

En esta última pregunta se observa una gran evolución en el aprendizaje, pasando de un $94,44 \%$ de los estudiantes con un total desconocimiento inicial de la cuestión a un $83,33 \%$ con la misma totalmente asimilada. Un escaso porcentaje aparece en un nuevo escalón intermedio (5,55\%), con respuestas en las que se aprecia cómo no han aprendido a diferenciar los métodos de cálculo a utilizar según la viga sea isostática o hiperestática.

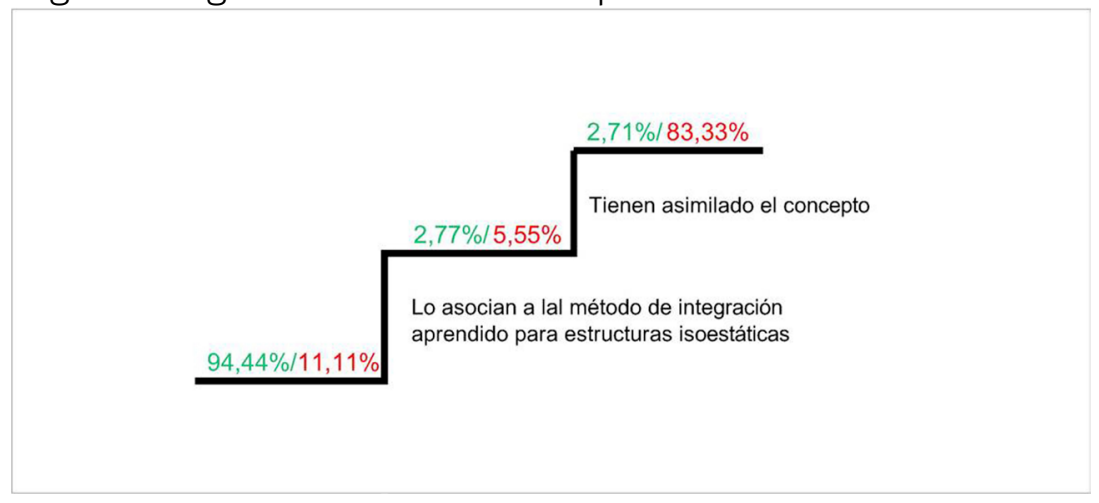

Figura 6. Escalera de aprendizaje pregunta 4

Jornadas de Formación e Innovación Docente del Profesorado | № 1 (2018)

(i) Esta obra se distribuye con la licencia Creative Commons

Reconocimiento-NoComercial-SinObraDerivada

4.0

Internacional (CC BY-NC-ND 4.0.) 


\section{Evaluación del diseño puesto en práctica}

Respecto al modelo metodológico diseñado en el aula, comentar que ha sido bastante acertado, siendo muy aceptable el nivel de implicación de los estudiantes. Sin embargo, el excesivo número de grupos (en torno a 20) ha tenido como consecuencias que el tiempo programado para cada actividad se haya alargado respecto a la programación inicial al no poder atenderlos a todos en ese período y que algunos alumnos hayan pasado desapercibidos en los debates y que éstos hayan sido más complicados de moderar, alargándose en consecuencia más tiempo del inicialmente previsto. A pesar de ello, se ha observado una importante evolución de los estudiantes en las sucesivas exposiciones y debates a nivel de pérdida de miedo a hablar en público, no así en su capacidad para expresar sus razonamientos correctamente. Respecto a este último aspecto, opino que sería necesario realizar más clases con esta metodología para mejorar esta parcela, fundamental en su carrera profesional.

En cuanto a los contenidos incluidos en el mapa (Figura 2), la estructura de las Hojas de Trabajo ha permitido tratarlos todos de manera progresiva y lógica, comenzando siempre con un proceso de razonamiento escalonado que les ha permitido llegar al entendimiento de un determinado concepto para después ponerlo en práctica a través de una actividad de carácter procedimental.

El resultado obtenido en las escaleras de aprendizaje refleja que un alto porcentaje de alumnos ha asimilado gran parte de los contenidos conceptuales, muy próximo al $100 \%$ en las cuestiones 3 y 4 .

Debido a que el ciclo se ha realizado entre finales de octubre y principios del mes de noviembre del curso actual, no se ha podido incluir en esta comunicación los resultados obtenidos tras la realización por parte de los estudiantes de los ejercicios individuales (trabajo "Fuera del aula" en modelo metodológico). 


\section{Conclusiones finales}

Partiendo de una valoración muy positiva de esta experiencia, como conclusiones finales cabe destacar:

- El trabajo en el aula permite el acercamiento profesor-alumno y aporta al docente una visión diferente de la enseñanza. El asesoramiento individual es una manera de enseñar mucho más directa y personalizada que, además de ser muy gratificante, resulta mucho más eficaz para ambas partes.

- La metodología puesta en práctica ayuda sobremanera al profesor a la hora del planteamiento de sus explicaciones colectivas. El conocimiento de las debilidades y fortalezas individuales de cada estudiante permite orientar sus exposiciones hacia la resolución de las mismas.

- El acercamiento estudiante-profesor permite un conocimiento mutuo que no se consigue en las clases magistrales, teniendo como consecuencia que el estudiante le plantee sus dudas sin ningún tipo de reservas y con absoluta confianza.

- La realización de los mapas de contenidos y preguntas clave resulta de gran ayuda al docente en el planteamiento de las clases y la estructuración del aprendizaje de los estudiantes.

- La puntuación de trabajos durante el período de impartición de la asignatura dentro de un sistema de evaluación continua estimula al estudiante y opino, a título individual, que es una metodología acertada para establecer los criterios de evaluación de las asignaturas.

- La implantación de esta metodología en más temas de la asignatura permitiría mejorar aspectos como la capacidad de concreción y de comunicación tanto oral como escrita de los estudiantes.

- Es necesaria la concienciación de los dirigentes universitarios de que un salto de calidad en la docencia lleva aparejado una política de reducción de estudiantes 
